Since $g_{2}=0, g_{3}=1$, it is easy to prove inductively that $C_{n}=0$ unless $n \equiv 0$ modulo 3. Substitution in (2) shows that $z f(z)$ is a function of $z^{3}$, so that

$$
f(\eta z)=\eta^{2} f(z), \quad \eta^{3}=1 .
$$

This shows that $f\left(-\eta^{2} h(z)\right)=\eta f(-h(z))$, and the proof of the equivalence of the various expressions for $G$ in (5) is complete.

\title{
REFERENCES
}

1. F. Gross, On the equation $f^{n}+g^{n}=1$, Bull. Amer. Math. Soc. 72 (1966), 86-88.

2. A. Hurwitz and R. Courant, Funktionentheorie, 4 Auflage, Springer, Berlin, 1964.

Imperial College of Science and Technology, London

\section{ON THE BOUNDARY BEHAVIOR OF FUNCTIONS MEROMORPHIC IN THE UNIT DISK}

\section{PETER COLWELL}

1. Introduction. Let $f(z)$ be meromorphic in $D:\{|z|<1\}$, and suppose that the values assumed by $f(z)$ in $D$ lie in a domain $G$ whose boundary $\Gamma$ has positive logarithmic capacity. Then $f(z)$ is of bounded characteristic in $D$ and has finite radial limits $f\left(e^{i \theta}\right)=\lim _{r \rightarrow 1} f\left(r e^{i \theta}\right)$ at almost all points $e^{i \theta}$ on $C:\{|z|=1\}$. (For this and more general theory of meromorphic functions, see [4, pp. $208 \mathrm{ff}$.].) The class of functions satisfying these conditions and having the additional property that $f\left(e^{i \theta}\right)$ belongs to $\Gamma$ almost everywhere on $C$ has been studied by O. Lehto [3] and D. A. Storvick [6], who called it class (L).

If $A$ is a sequence of points in $D$ satisfying $\sum_{a \in A}(1-|a|)<\infty$, the Blaschke product with respect to $A$ in $D$ is the function $B(z ; A)$ $=\prod_{a \in A}[|a|(a-z) / a(1-\bar{a} z)]$. The present note arises from a suggestion by Professor Storvick that the following theorem, established in $[1]$, be extended to functions in class (L). Here we denote by $A^{\prime}$ the derived set of $A$.

Theorem 1. Let $E$ be a set on $C$. A necessary and sufficient condition

Presented to the Society, November 5, 1965; received by the editors February $17,1966$. 
that there exist a Blaschke product $B(z ; A)$ for which $B\left(e^{i \theta}\right)$ $=\lim _{r \rightarrow 1} B\left(r e^{i \theta} ; A\right)$ is defined and of modulus one at every point of $C$ and such that $A^{\prime}=E$ is that $E$ be closed and nowhere dense on $C$.

2. Let $G$ be a domain whose boundary $\Gamma$ has positive logarithmic capacity.

THEOREM 2. Let $f(z)$ be a function of class (L) with respect to $G$ and $\Gamma$, and suppose that $f\left(e^{i \theta}\right)=\lim _{r \rightarrow 1} f\left(r e^{i \theta}\right)$ exists and belongs to $\Gamma$ for every $e^{i \theta}$ on $C$. Then if $a$ is any point of $G$ and $A=\{z \in D: f(z)=a\}$, $A^{\prime}$ is closed and nowhere dense on $C$.

We note first that $A$ is not empty, since O. Lehto [3, p. 12] showed that any omitted value of $f$ in $G$ is a radial limit for $f(z)$. Also from $[3$, p. 12], if $A$ is finite then $a$ must be a boundary value for $f(z)$, unless $G$ is simply-connected and $f(z)=\rho[R(z)]$ for $R(z)$ rational, $|R(z)| \leqq 1,\left|R\left(e^{i \theta}\right)\right|=1$, and $w=\rho(z)$ a schlicht function mapping $D$ onto $G$. In the latter case $f(z)$ assumes each of its values only finitely many times, and the theorem is clearly true.

For the case when $A$ is infinite, $A^{\prime} \cap D=\varnothing$ since $f(z)$ is meromorphic in $D$, and $A^{\prime}$ is closed. For any point $e^{i \theta}$ of $A^{\prime}, f\left(e^{i \theta}\right)$ $=\lim _{r \rightarrow 1} f\left(r e^{i \theta}\right)$ is a point $\gamma(\theta)$ of $\Gamma$. That is, the radial cluster set, $C_{r}\left(f, e^{i \theta}\right)$, for $f$ at $e^{i \theta}$ is a single point $\gamma(\theta)$. However, the interior cluster set, $C_{D}\left(f, e^{i \theta}\right)$, for $f$ at $e^{i \theta}$ contains at least the points $a$ and $\gamma(\theta)$. Thus for $e^{i \theta}$ in $A^{\prime}$ we have $C_{D}\left(f, e^{i \theta}\right) \neq C_{r}\left(f, e^{i \theta}\right)$. By a theorem of E. F. Collingwood [2, p. 378], $A^{\prime}$ must be a set of category $I$ on $C$. Since $A^{\prime}$ is closed, $A^{\prime}$ is necessarily nowhere dense on $C$. (The method of proof that $A^{\prime}$ is nowhere dense on $C$ was originally suggested to the author by Professor K. Noshiro for use in the proof of Theorem 1.)

3. In the special case that $G$ is simply-connected and its boundary $\Gamma$ is a Jordan curve, we prove the following

THEOREM 3. Let a be any point of a simply-connected domain $G$ whose boundary $\Gamma$ is a Jordan curve. Let $E$ be a closed nowhere dense set on $C$. Then there exists a function, analytic in $D$, such that: (i) $f(z)$ assumes its values in $G$; (ii) for every $e^{i \theta}$ on $C$ the limit $\lim _{r \rightarrow 1} f\left(r e^{i \theta}\right)=f\left(e^{i \theta}\right)$ exists and belongs to $\Gamma$; (iii) if $A=\{z \in D: f(z)=a\}$, then $A^{\prime}=E$.

If $\rho=g(w)$ is a conformal mapping of $G$ onto $\{|\rho|<1\}$ with $g(a)$ $=0, g^{\prime}(a)>0$, then $g$ can be extended to a homeomorphism of $G \cup \Gamma$ onto $\{|\rho| \leqq 1\}$, and we can consider the mapping $w=g^{-1}(\rho)$ as a homeomorphism of $\{|\rho| \leqq 1\}$ onto $G \cup \Gamma$ which is analytic in $\{|\rho|<1\}$ and assumes values there in $G$.

By Theorem 1 , since $E$ is closed and nowhere dense on $C$, there 
exists a Blaschke product $B(z ; H)$ in $D$ with radial limits of modulus one at every point of $C$ such that $H^{\prime}=E$. (Here $H$ is the set of zeros for $B(z ; H)$ in $D$, and $\sum_{h \in H}(1-|h|)<\infty$.) We let $\rho=B(z ; H)$ and define $w=g^{-1}[B(z ; H)]$ for $z$ in $D$.

The image under $B(z ; H)$ of $D$ is the disk $\{|\rho|<1\}$, since W. Seidel [5] showed that any omitted value in $\{|\rho|<1\}$ is a radial limit value for $B(z ; H)$ at some point of $C$. It is easily verified that $w=f(z)$ is analytic in $D$ and satisfies (i), (ii), and (iii).

\section{BIBLIOGRAPHY}

1. P. Colwell, On the boundary behavior of Blaschke products in the unit disk, Proc. Amer. Math. Soc. 17 (1966), 582-587.

2. E. F. Collingwood, On the sets of maximum indetermination of analytic functions, Math. Z. 67 (1957), 377-396.

3. O. Lehto, On meromorphic functions whose values lie in a given domain, Ann. Acad. Sci. Fenn. A I 160 (1953), 1-14.

4. R. Nevanlinna, Eindeutige Analytische Funktionen, Springer, Berlin, 1953.

5. W. Seidel, On the distribution of values of bounded analytic functions, Trans. Amer. Math. Soc. 36 (1934), 201-226.

6. D. A. Storvick, On meromorphic functions of bounded characteristic, Proc. Amer. Math. Soc. 8(1957), 32-38.

Iowa State University 